\title{
Comparação entre filmes radiográficos e o sistema digital Digora, no diagnóstico de lesões de cárie dentinária em superfície proximal de molares decíduos ${ }^{\dagger}$
}

\section{Comparison between radiographic films and the Digora digital system in the diagnosis of approximal dentinal caries in primary molars}

\author{
Maximiano Ferreira TOVO* \\ Bernardo Gonzalez VONO** \\ Orivaldo TAVANO***
}

\begin{abstract}
TOVO, M. F.; VONO, B. G.; TAVANO, O. Comparação entre filmes radiográficos e o sistema digital Digora, no diagnóstico de lesões de cárie dentinária em superfície proximal de molares decíduos. Pesqui Odontol Bras, v. 14, n. 4, p. 399-405, out./dez. 2000.
\end{abstract}

\begin{abstract}
O estudo comparou o desempenho diagnóstico dos filmes Agfa M2 e Ektaspeed Plus e do sistema digital Digora. A amostra foi composta por 37 molares decíduos, radiografados em 50, 70 e $90 \mathrm{kV}$. As imagens de 41 superficies proximais foram avaliadas sob condições padronizadas. Cinco recursos de edição de imagem (0,5 X, 1 X, 2 X, "Negativo" e 3D), disponiveis no software do sistema digital, foram utilizados. Após a secção dos dentes e a validação em estereomicroscopia $(40 \mathrm{X})$, calculou-se a sensibilidade, especificidade, acurácia e kappa não-ponderado dos métodos. O filme Ektaspeed Plus, exposto a $50 \mathrm{kV}$, diferenciou-se devido aos valores expressos para sensibilidade, acurácia e kappa não-ponderado. A especificidade dos filmes revelou valores comparáveis. Com a utilização do sistema digital Digora, a taxa de diagnósticos positivo-verdadeiros alcançou maior valor ao ser utilizado o recurso de imagem "Negativo". A imagem comprimida $(0,5 \mathrm{X})$ foi, majoritariamente, a mais específica. As acurácias médias do sistema digital Digora resultaram em valores aproximados. Ao ser utilizada a estatística kappa, não houve distinção entre os recursos de imagem obtidos com o software. O método digital foi mais sensivel que os filmes, porém, estes foram mais específicos. A acurácia média do sistema digital suplantou a dos filmes. Excetuando-se o Ektaspeed Plus, em $50 \mathrm{kV}$, os filmes não alcançaram os valores de kappa demonstrados pelo sistema digital Digora. Cohen's kappa revelou boa concordância intra-examinador $(0,758)$. Devido aos resultados observados, o sistema digital demonstrou-se comparável ao método radiográfico convencional, no diagnóstico de lesões de cárie dentinária em superfícies proximais de molares decíduos.
\end{abstract}

UNITERMOS: Cárie dentária; Diagnóstico por computador; Filme para raios X; Dente decíduo.

\section{INTRODUÇÃO}

A imagem radiográfica interproximal permite condição informativa diferenciada e indispensável para a avaliação do paciente, quando perscrutam-se lesões da doença cárie localizadas em superfícies dentárias contíguas, inacessiveis ao diagnóstico clínico visual.

Ao abordar este tema, a Radiologia Odontológica demonstra-se essencialmente bioética, com a busca incessante de conhecimentos que culminem em uma menor dose de radiação aplicada ao paci- ente aliada à qualidade dos dados obtidos. Exemplo deste propósito é o desenvolvimento de filmes de sensibilidade crescente, a despeito da inexorável e tão discutida perda da nitidez da imagem $^{8,9,13,15,23}$. Nas duas últimas décadas, ao incorporar recursos da computação digital, a Radiologia Odontológica demonstrou uma notável diferenciação tecnológica e potencializou sua condição de um importante recurso diagnóstico auxiliar ${ }^{25,27,28}$.

Atualmente, as imagens radiográficas podem ser obtidas por métodos digitais diretos, os quais dispensam a utilização de filmes ${ }^{11,13,16,17,19}$. Estudado

\footnotetext{
${ }^{\dagger}$ Parte da Tese de Doutorado (Odontopediatria) apresentada à Faculdade de Odontologia de Bauru da USP.

* Mestre e Doutor em Odontopediatria; ** Professor Titular do Departamento de Odontopediatria e Ortodontia; *** Professor Titular do Departamento de Estomatologia - Faculdade de Odontologia de Bauru da USP.
} 
TOVO, M. F.; VONO, B. G.; TAVANO, O. Comparação entre filmes radiográficos e o sistema digital Digora, no diagnóstico de lesões de cárie dentinária em superfície proximal de molares decíduos. Pesqui Odontol Bras, v. 14, n. 4, p. 399-405, out./dez. 2000 .

em recentes pesquisas ${ }^{3,4,10,11,12,18,19,22,26,27,30}$, o sistema digital Digora apresenta uma imagem composta por vários pontos (pixels) com diferentes tonalidades de cinza, resultante da leitura a laser de uma placa óptica exposta à radiação $\mathrm{X}^{5}$.

São incipientes os estudos nacionais que tenham por objetivo avaliar o diagnóstico de lesões de cárie por métodos digitais ${ }^{10,14}$. Em especial, ao tratar-se de uma amostra composta por dentes decíduos, acentua-se a escassez de informações, até mesmo quando recorremos a publicações internacionais $^{19}$.

Diante do exposto, um estudo que possibilite avaliar a capacidade diagnóstica dos métodos utilizados para detecção de lesões dentinárias em superficies proximais de molares decíduos, por meio do exame radiográfico com filmes e digital, contribuirá para uma melhor compreensão dos fatos citados.

\section{MATERIAL E MÉTODOS}

Foram examinadas 41 superficies proximais de 37 molares decíduos extraídos, os quais foram radiografados em três quilovoltagens. Os dentes foram posicionados dois a dois no filme radiográfico por meio de blocos de cera, sobre uma bancada auxiliar e submetidos às incidências radiográficas. $O$ raio central foi dirigido para o centro do filme radiográfico, onde localizavam-se as coroas dentárias. O ângulo horizontal orientou o feixe central dos raios $\mathrm{X}$ de forma que ele estivesse paralelo às faces proximais dos dentes, e o ângulo vertical foi posicionado em $90^{\circ}$. A incidência deu-se no sentido vestíbulo-lingual ${ }^{1}$. Para padronizar a técnica, foi utilizado um porta-filme com anel localizador longo acoplado e a distância anódio/filme definida em $35 \mathrm{~cm}$. Ao ser utilizado o aparelho Spectro II (Dabi Atlante) de $50 \mathrm{kVp}, 10 \mathrm{~mA}$, o tempo de exposição definido para o filme Ektaspeed Plus* foi $6 \mathrm{mAs}$; para o filme Agfa Dentus M2**, 7 mAs. Para o aparelho Spectro $70 \mathrm{X}$ (Dabi Atlante), de $70 \mathrm{kVp}$, $8 \mathrm{mAs}$, o tempo de exposição para o filme Ektaspeed Plus foi 3,2 mAs; para o filme Agfa Dentus M2, $3,6 \mathrm{mAs}$. Ao aparelho GE 100, regulado para $90 \mathrm{kVp}, 10 \mathrm{~mA}$, o tempo de exposição foi $1,5 \mathrm{mAs}$ para o filme Ektaspeed Plus e $2 \mathrm{mAs}$ para o filme Agfa Dentus M2. A análise radiográfica realizada por um examinador calibrado seguiu os critérios:
0 - ausência de radiotransparência; 1 - radiotransparência em esmalte, aquém da metade mais externa de sua espessura; 2 - radiotransparência em esmalte, na metade mais interna de sua espessura, sem atingir o limite amelodentinário; 3 - radiotransparência em esmalte, atingindo o limite amelodentinário; 4 - radiotransparência em dentina, limitando-se à metade mais externa de sua espessura; 5 - radiotransparência em dentina, na metade mais interna de sua espessura.

As incidências obtidas com o sistema digital Digora seguiram a metodologia aplicada ao exame radiográfico convencional. As placas de imagem utilizadas possuíam a dimensão de $35 \mathrm{~mm} \mathrm{x}$ $45 \mathrm{~mm} \times 1,6 \mathrm{~mm}$ e foram protegidas da luz ambiente com o vedamento do invólucro plástico original. A dose de exposição aos raios X aplicada às placas seguiu a recomendação do fabricante, ou seja, 20 a 50\% da dose utilizada em filmes de radiografia ${ }^{5}$. Com base nessa orientação, as placas, ao serem utilizadas no aparelho de $50 \mathrm{kVp}$, foram submetidas a $3 \mathrm{mAs}$ de incidência; no aparelho de $70 \mathrm{kVp}$, a 1,6 mAs e, no de $90 \mathrm{kVp}$, a 0,8 mAs. As imagens foram observadas operando o software Digora for Windows versão 1.51, nas seguintes funções: redução da imagem à metade de seu tamanho original (representada, no programa, pelo botão - ou ícone - "0,5"); imagem no tamanho original (icone "1"); tamanho duplicado da imagem (icone "2"); visualização da imagem em negativo (icone representando um retângulo com uma metade escura e a outra clara) e a imagem tridimensional (ícone "3D"). Nos modos "Negativo" e "3D", as imagens foram dispostas no monitor na dimensão original (icone "1"). No intuito de otimizar as imagens, foram utilizados os ajustes de contraste e luminosidade disponiveis no programa. Os critérios adotados para definir a presença de lesão de cárie nas superficies proximais, ao serem analisadas as imagens digitais, foram os mesmos do exame radiográfico convencional. A análise das imagens geradas pelo sistema Digora, na tela do monitor, obedeceu a uma padronização semelhante à do exame radiográfico convencional, de forma que ela se repetiu sob turnos programados, jornadas distintas e em local com iluminação controlada.

Para a avaliação da presença ou ausência da lesão, os 41 sítios das superficies proximais foram

\footnotetext{
* Ektaspeed Plus EP 21P - Speed Group E - Eastman Kodak Company, EUA.

** Agfa Dentus M2 Comfort Dental Film - Speed Group D - Agfa Gevaert N. V., Bélgica.
} 
TOVO, M. F.; VONO, B. G.; TAVANO, O. Comparação entre filmes radiográficos e o sistema digital Digora, no diagnóstico de lesões de cárie dentinária em superfície proximal de molares decíduos. Pesqui Odontol Bras, v. 14, n. 4, p. 399-405, out./dez. 2000 .

seccionados no sentido mesiodistal, com auxílio de um disco diamantado dupla face acoplado à máquina de corte, para posterior análise em estereomicroscópio com luz incidente, com aumento de 40 vezes. Os sítios foram registrados seguindo os critérios assim definidos: 0 - ausência de lesão; 1 lesão de cárie em esmalte, aquém da metade mais externa de sua espessura; 2 - lesão de cárie em esmalte, na metade mais interna de sua espessura, sem atingir o limite amelodentinário; 3 - lesão de cárie em esmalte, atingindo o limite amelodentinário; 4 - lesão de cárie em dentina, limitando-se à metade mais externa de sua espessura; 5 - lesão de cárie em dentina, na metade mais interna de sua espessura.

A análise microscópica das áreas seccionadas foi utilizada para validar os dentes corretamente diagnosticados. Pelo cotejamento dos resultados do "gold standard" (análise microscópica) com os expressos pelos métodos em estudo, construiu-se a matriz de decisões (Tabela 1). Esta, ao revelar as quatro possibilidades de decisões (positivo-verdadeiras, falso-positivas, negativo-verdadeiras e falso-negativas), permitiu o cálculo das proporções utilizadas neste estudo: sensibilidade $(P V / P V+F N)$, especificidade $(N V / N V+F P)$ e acurácia $(P V+N V / P V+$ $F N+N V+F P)$. A concordância entre os métodos estudados e o "gold standard" também foi calculada pela estatística kappa $(k)$.

Com o intuito de analisar a reprodutibilidade diagnóstica, foi calculada a variação intra-examinador. O cálculo foi realizado mediante o emprego do software Kappa Solver versão 0.1b97*. Os valores de $k$ expressando a concordância entre os métodos e o padrão, bem como a reprodutibilidade, obedeceram à escala do Cohen's kappa.

\section{RESULTADOS}

Na Tabela 2, a correlação entre o exame clínico visual macroscópico - o qual classificou os sítios

TABELA 1 - Matriz de decisões.

\begin{tabular}{l|c|c}
\hline \hline \multirow{2}{*}{$\begin{array}{c}\text { Resultado } \\
\text { do teste }\end{array}$} & Presente & Doença \\
\cline { 2 - 3 } Positivo & Positivo-verdadeiro & Falso-positivo \\
\hline Negativo & Falso-negativo & Negativo-verdadeiro \\
\hline \hline
\end{tabular}

Fonte: DOUGLAS; McNEIL6. proximais em hígido, com mancha branca e com cavidade - e a análise microscópica, a qual classificou os referidos sítios de hígidos a portadores de lesão dentinária profunda pode ser verificada.

Em valores percentuais, a sensibilidade do filme radiográfico Agfa Dentus M2, nas diferentes quilovoltagens $(50,70$ e $90 \mathrm{kV})$, foi 55 , 50 e $65 \%$. A especificidade, na mais baixa e média quilovoltagem atingiu 100\%; na maior, 87,5\%. A acurácia, na quilovoltagem média e na mais alta, foi de 75,6\%; em $50 \mathrm{kV}, 78,04 \%$.

O filme Ektaspeed Plus apresentou valores percentuais de sensibilidade decrescentes, à medida que se aumentava a quilovoltagem (65, 55 e 50\%). A especificidade nas quilovoltagens extremas foi $100 \%$ e em $70 \mathrm{kV}, 95,2 \%$. A acurácia foi $82,92 \%$ em $50 \mathrm{kV}$; nas demais quilovoltagens, manteve o mesmo valor: $75,6 \%$.

Os valores de sensibilidade, especificidade e acurácia do sistema digital Digora, levando-se em consideração os diferentes recursos para manipulação de imagem empregados e as diferentes quilovoltagens, estão expressos nas Tabelas 3, 4, e 5. A acurácia média do sistema digital foi de mesmo valor em 50 e $90 \mathrm{kV}: 84,38 \%$; em $70 \mathrm{kV}$, foi de $84,87 \%$.

Ao considerarmos os valores de kappa $(k)$ não-ponderado na validação dos resultados, foi observado que em 70 e $90 \mathrm{kV}$ os dois filmes estudados apresentaram valores aproximados

TABELA 2 - Correlação entre o exame clínico visual (macroscópico) dos sítios proximais e a análise microscópica.

\begin{tabular}{l|c|c|c|c}
\hline \hline \multirow{2}{*}{$\begin{array}{c}\text { Análise } \\
\text { microscópica }\end{array}$} & \multicolumn{4}{|c}{ Exame clínico visual } \\
\cline { 2 - 5 } & Hígido & $\begin{array}{c}\text { Mancha } \\
\text { branca }\end{array}$ & Cavidade & Total \\
\hline Hígido & 5 & - & - & 5 \\
\hline Esmalte 1 & - & 4 & - & 4 \\
\hline Esmalte 2 & - & 5 & 2 & 7 \\
\hline Esmalte 3 & - & 2 & 3 & 5 \\
\hline Dentina 4 & - & $4 *$ & $9 * *$ & 13 \\
\hline $\begin{array}{l}\text { Dentina 5 } \\
\text { Total }\end{array}$ & - & - & 7 & 7 \\
\hline *3/4 (75\%) com pigmentação. ** 2/9 (22,2\%) com \\
pigmentação.
\end{tabular}

* Desenvolvido por MITTMANN, B.; FREITAS, S. F. T. - Pós-Graduação em Saúde Pública, UFSC. 
TOVO, M. F.; VONO, B. G.; TAVANO, O. Comparação entre filmes radiográficos e o sistema digital Digora, no diagnóstico de lesões de cárie dentinária em superfície proximal de molares decíduos. Pesqui Odontol Bras, v. 14, n. 4, p. 399-405, out./dez. 2000 .

$(k=0,5060$ a 0,5096$)$, sendo classificados na mesma escala qualitativa (regular). Em $50 \mathrm{kV}$, o filme Agfa manteve-se na classificação qualitativa regular $(k=0,5560)$ e o filme Ekta, ao resultar em $k=0,6555$, foi considerado de boa validação na escala qualitativa.

Ao considerarmos os valores de kappa $(k)$, não-ponderado no cotejamento dos resultados do sistema Digora com o "gold standard", a escala qualitativa categorizou a imagem digital como de boa validação, nas três quilovoltagens estudadas. Numericamente, os valores de $k$ variaram de 0,6058 a 0,7551 em $50 \mathrm{kV}$, de 0,6563 a 0,7557 em $70 \mathrm{kV}$ e, em $90 \mathrm{kV}$, de 0,6563 a 0,7551, de acordo com o recurso de imagem empregado.

A reprodutibilidade intra-examinador, avaliada pelo kappa $(k)$ não-ponderado, resultou em 0,758, sendo os exames considerados de boa confiabilidade.

\section{DISCUSSÃO}

Neste trabalho, não nos propusemos a caracterizar o grau de desmineralização das lesões, no entanto, ao observarmos as manchas brancas e as

TABELA 3 - Valores percentuais de sensibilidade, especificidade e acurácia do sistema digital Digora, em $50 \mathrm{kV}$.

\begin{tabular}{l|c|c|c}
\hline \hline $\begin{array}{c}\text { Recursos de } \\
\text { imagem }\end{array}$ & Sensibilidade & Especificidade & Acurácia \\
\hline $0,5 \mathrm{X}$ & 60 & 100 & 80,48 \\
\hline $1 \mathrm{X}$ & 80 & 95 & 87,80 \\
\hline $2 \mathrm{X}$ & 75 & 95 & 85,36 \\
\hline Negativo & 75 & 95 & 85,36 \\
\hline $3 \mathrm{D}$ & 70 & 95 & 82,92 \\
\hline \hline
\end{tabular}

TABELA 4 - Valores percentuais de sensibilidade, especificidade e acurácia do sistema digital Digora, em $70 \mathrm{kV}$.

\begin{tabular}{l|c|c|c}
\hline \hline $\begin{array}{c}\text { Recursos de } \\
\text { imagem }\end{array}$ & Sensibilidade & Especificidade & Acurácia \\
\hline $0,5 \mathrm{X}$ & 70 & 95 & 82,92 \\
\hline $1 \mathrm{X}$ & 80 & 95 & 87,80 \\
\hline $2 \mathrm{X}$ & 70 & 95 & 82,92 \\
\hline Negativo & 85 & 90,47 & 87,80 \\
\hline 3D & 70 & 95 & 82,92 \\
\hline \hline
\end{tabular}

cavidades das lesões em dentina, foram denotadas algumas peculiaridades. Lesões dentinárias (escore 4), com mancha branca e sem cavidade, exibiam manchas acastanhadas, sugerindo, na área afetada, envolvimento em processo de remineralização. Das quatro lesões em dentina atingindo a metade da extensão tecidual, três apresentavam manchas brancas acastanhadas (Tabela 2).

Neste mesmo grau de profundidade, algumas lesões com cavidade também estavam pigmentadas (em esmalte). Foram encontradas, entre estas, $22,2 \%$ de cavidades pigmentadas (duas, entre nove) (Tabela 2).

Conforme revelaram WENZEL et al. ${ }^{29}$, estes aspectos podem influenciar na sensibilidade diagnóstica de um método em avaliação. Podem, também, explicar a variação de resultados das pesquisas, mesmo entre os trabalhos que adotam similares niveis de profundidade microscópica da lesão como padrão para validar os métodos. Salvo a referência citada, os trabalhos não discutem esta variável, quando refletem sobre a capacidade diagnóstica dos métodos, fato que, a nosso ver, deve ser considerado. A leitura atenta fez-nos concluir que, em algumas publicações, tais alterações estão registradas como característica da amostra; no entanto, suas participações nos resultados passam despercebidas ${ }^{20}$. O comportamento radiográfico destas lesões ainda não foi elucidado pela literatura. Em nosso estudo, no diagnóstico de lesões dentinárias, todos os resultados falso-negativos, sem exceção nenhuma, envolveram as lesões que não se encontravam profundamente neste tecido (escore 4).

A taxa de diagnósticos falso-negativos foi alta nos dois filmes estudados; a de falso-positivos, nula, na maioria das vezes. Interpretamos nossos resultados com a mesma inferência desenvolvida

TABELA 5 - Valores percentuais de sensibilidade, especificidade e acurácia do sistema digital Digora, em $90 \mathrm{kV}$.

\begin{tabular}{l|c|c|c}
\hline $\begin{array}{c}\text { Recursos de } \\
\text { imagem }\end{array}$ & Sensibilidade & Especificidade & Acurácia \\
\hline $0,5 \mathrm{X}$ & 65 & 100 & 82,92 \\
\hline $1 \mathrm{X}$ & 75 & 95 & 85,36 \\
\hline $2 \mathrm{X}$ & 70 & 95 & 82,42 \\
\hline Negativo & 80 & 95 & 87,80 \\
\hline 3D & 70 & 95 & 82,92 \\
\hline \hline
\end{tabular}


TOVO, M. F.; VONO, B. G.; TAVANO, O. Comparação entre filmes radiográficos e o sistema digital Digora, no diagnóstico de lesões de cárie dentinária em superfície proximal de molares decíduos. Pesqui Odontol Bras, v. 14, n. 4, p. 399-405, out./dez. 2000 .

por RICKETTS et $a .^{21}$, para os quais a reduzida sensibilidade foi explicada com base na presença de considerável proporção de lesões dentinárias superficiais na amostra estudada. A alta especificidade, para os referidos autores, isenta os métodos estudados de acusar diagnósticos falso-positivos para lesões em dentina, fato também observado por nós. O aumento da especificidade é esperado quando se define a presença de lesão dentinária, pois a probabilidade de diagnósticos falso-positivos, nesta profundidade, é reduzida. O incremento da especificidade também constou nos resultados de VERDONSCHOT et al. ${ }^{24}$, assim como foram altos para RICKETTS et $a l^{21}$.

Nos valores de acurácia para o diagnóstico de lesões dentinárias, as diferenças numéricas entre os filmes, nas respectivas quilovoltagens, foram reduzidas. Para o filme Agfa, variou de 75,6 a $78,04 \%$, no intervalo de 50 a $70 \mathrm{kV}$. Para o Ekta, nas mesmas quilovoltagens, variou de 75,6 a $82,92 \%$, sendo este resultado considerado bom, perante a validação da estatística kappa. Pelos valores demonstrados, os filmes, nas diferentes quilovoltagens, foram considerados de capacidade diagnóstica comparável, na detecção de lesões dentinárias em superficies proximais de molares decíduos.

A sensibilidade do sistema radiográfico digital sofreu variações de acordo com o recurso de imagem e com a quilovoltagem empregada. Em geral, a taxa de diagnósticos positivo-verdadeiros foi alta e a porcentagem de falso-negativos reduzida (Tabelas 3, 4 e 5), o que favoreceu os índices de sensibilidade. A inversão do contraste (imagem em "Negativo"), em $70 \mathrm{kV}$, revelou a maior taxa de positivo-verdadeiros.

No tocante à confirmação de ausência de lesão, a especificidade esteve comprometida. Por vezes, houve diagnósticos falso-positivos, o que reduziu a taxa de diagnósticos negativos corretamente afirmados. Este resultado configurou-se na redução da especificidade, visto que há relação inversa entre a taxa de falso-positivos e negativo-verdadeiros (Tabelas 3, 4 e 5). A imagem comprimida $(0,5 \mathrm{X})$ revelou, em duas quilovoltagens (50 e $90 \mathrm{kV}$ ), os mais altos valores de especificidade.

A pequena variação numérica da acurácia média do sistema digital, em relação às quilovoltagens, foi confirmada pelos valores do kappa não-ponderado na validação do diagnóstico de lesões proximais. A variação da dose de radiação X aplicada às placas ópticas não determinou diferenças diagnósticas a ponto de justificar a eleição de uma ou outra dosagem. Independentemente da quilovoltagem, o comportamento do método, numérica e qualitativamente expresso, tende a ser comparável. FARMAN ${ }^{7}$, ao avaliar as propriedades técnicas de um sistema digital direto, submeteu as incidências à variação de quilovoltagem (50 a $90 \mathrm{kV}$ ) e considerou praticamente idênticas as respostas da qualidade da imagem à dose.

Este dado é de suma importância, posto que algumas publicações não consideram os valores de quilovoltagem na reciprocidade dos dados discuti$\operatorname{dos}^{17,20,24}$. Por nossos resultados, consideramos viável a comparação da acurácia diagnóstica entre experimentos que façam uso de diferentes quilovoltagens, quando é utilizado o sistema digital.

A imagem comprimida pelo sistema Digora $(0,5 \mathrm{X})$, dentre as modalidades de imagem digital avaliadas, revelou a menor sensibilidade, em todas as quilovoltagens. Esta modalidade possui dimensões semelhantes às do filme, sendo esta a provável explicação para o resultado obtido. O diagnóstico das lesões dentinárias pareceu-nos não se beneficiar do recurso de imagem citado.

Pode-se depreender que a principal diferença entre os métodos estudados nesta pesquisa (sistema digital e filme convencional), quanto à validação do diagnóstico de lesões dentinárias de superficies proximais de molares decíduos, repousa na taxa de positivo-verdadeiros (sensibilidade do teste). Como as diferenças desta proporção foram maiores que as observadas entre as especificidades, a acurácia do sistema Digora sobrepujou a dos filmes, em média. As condições otimizadas de contraste obtidas pelo sistema digital podem ter influenciado a detecção de lesões, visto que os filmes apresentaram menor sensibilidade. A definição dos mesmos, ainda que referida como superior, provavelmente foi subjugada pelos recursos de imagem disponiveis no software, sendo esta premissa compativel com o postulado por BENN ${ }^{2}$.

\section{CONCLUSÕES}

1. Em relação aos filmes radiográficos: o filme Ektaspeed Plus, exposto a $50 \mathrm{kV}$, demonstrou o maior valor de acurácia e sensibilidade, sendo esta igual ao do filme Agfa M2 em $90 \mathrm{kV}$; a especificidade dos filmes revelou valores comparáveis. 
TOVO, M. F.; VONO, B. G.; TAVANO, O. Comparação entre filmes radiográficos e o sistema digital Digora, no diagnóstico de lesões de cárie dentinária em superfície proximal de molares decíduos. Pesqui Odontol Bras, v. 14, n. 4, p. 399-405, out./dez. 2000 .

2. Em relação ao sistema Digora: o maior valor de sensibilidade foi revelado pela modalidade de imagem "Negativo" (70 kV); majoritariamente, a imagem em 0,5 X foi a mais específica $(50 \mathrm{e}$ $90 \mathrm{kV}$ ). A acurácia média do sistema digital Digora foi, em valores, praticamente a mesma e a estatística kappa resultou em validade boa.
3. Comparando-se os filmes e o sistema Digora: o sistema digital Digora foi mais sensivel que os filmes, em todos os $\mathrm{kV}$, não ocorrendo o mesmo em relação à especificidade. A acurácia média do sistema digital suplantou a dos filmes e somente o filme Ekta, em $50 \mathrm{kV}$, alcançou os valores de kappa revelados pelo sistema digital.

TOVO, M. F.; VONO, B. G.; TAVANO, O. Comparison between radiographic films and the Digora digital system in the diagnosis of approximal dentinal caries in primary molars. Pesqui Odontol Bras, v. 14, n. 4, p. 399-405, out./dez. 2000.

This study evaluated the diagnostic performance of the Agfa M2 and Ektaspeed Plus dental films and the Digora digital system. The material comprised 37 extracted primary molars radiographed with 50, 70 and $90 \mathrm{kV}$. The images of 41 approximal surfaces were examined under standardized conditions. Five imaging modes $(0.5 \mathrm{X}, 1 \mathrm{X}, 2 \mathrm{X}$, "Negative" and 3D) available in the software of the digital system, were used. The teeth were sectioned and observed under a stereomicroscope $(40 \mathrm{X})$. The data were analysed for sensitivity, specificity, accuracy and unweighted kappa. Ektaspeed plus exposed to $50 \mathrm{kV}$ obtained the highest sensitivity, accuracy and kappa values. The specificity values of the films were comparable. Digora presented the highest values of sensitivity when the "Negative" mode was used. The majority of the reduced images $(0.5 \mathrm{X})$ revealed the highest specificity. The average accuracy values of the digital system were very similar. Unweighted kappa revealed that all digital image modes established good agreement. The sensitivity values of the digital system were higher than that of the conventional films, but films revealed higher values of specificity. The average accuracy values of Digora were higher than that of the films, for all kilovoltages. Except for Ektaspeed Plus at $50 \mathrm{kV}$, the films did not reach the kappa values of Digora in the diagnosis of dentinal caries. Cohen's kappa revealed good intra-examiner reliability (0.758). It was demonstrated that the digital system is comparable to the conventional radiographic method in the diagnosis of approximal dentinal caries in primary molars.

UNITERMS: Dental caries; Diagnosis, computer-assisted; X-ray film; Tooth, deciduous.

\section{REFERÊNCIAS BIBLIOGRÁFICAS}

1. AlVARES, L. C.; TAVANO, O. Curso de Radiologia em Odontologia. 3. ed. São Paulo : Santos. 1993.

2. BENN, D. K. Radiographic caries diagnosis and monitoring. Dentomaxillofac Radiol, v. 23, n. 2, p. 69-72, May 1994.

3. BORG, E.; GRÖNDAHL, H. G. Dynamic range of film and digital systems for image acquisition in intra-oral radiography. Dentomaxillofac Radiol, v. 24, n. 2, p. 104-105, May 1995.

4. CEDERBERG, R. A.; FREDERIKSEN, N. L.; BENSON, B. $\mathrm{W}$. et al. ROC analysis of digital and film radiographic images for the detection of approximal lesions. Dentomaxillofac Radiol, v. 27, n. 2, p. 119-120, Mar. 1998. (Abstract)

5. DIGORA: Manual do proprietário. Orion Corporation Soredex. Helsinki, Finland.

6. DOUGLASS, C. W.; McNEIL, B. J. Clinical decision analysis methods applied to diagnostic tests in Dentistry. $\mathbf{J}$ Dent Educ, v. 47, n. 11, p. 708-712, Nov. 1983.

7. FARMAN, A. G. Basic technical properties of a system for direct acquisition of digital intraoral radiographs. Oral Surg Oral Med Oral Pathol, v. 75, n. 4, p. 506-516, Apr. 1993.

8. FROMMER, H. H.; JAIN, R. K. A comparative clinical study of group D and E dental films. Oral Surg Oral Med Oral Pathol, v. 63, n. 6, p. 738-742, June 1987.
9. GHILARDI NETTO, T.; DE PAULA, E.; BAFFA, O. et. al. Características sensitométricas de filmes utilizados em radiologia odontológica. Odont Mod, v. 15, n. 2, p. 6-12, mar. 1988.

10. GONÇALVES, M. R. Estudo da efetividade de métodos de diagnóstico de lesões cariosas oclusais e da correlação entre os métodos de validação. Araçatuba, 1998. 145 p. Tese (Doutorado) - Faculdade de Odontologia de Araçatuba da Universidade Estadual Paulista.

11. GOTFREDSEN, E.; WENZEL, A.; GRÖNDAHL, H. G. Observer's use of image enhancement in assessing caries in radiographs taken by four intra-oral digital systems. Dentomaxillofac Radiol, v. 25, n. 1, p. 34-38, Jan. 1996.

12. GRÖNDHAL, H. G.; WENZEL, A.; BORG, E. et al. An image plate system for digital intra-oral radiography. Dent Update, v. 23, n. 8, p. 334-337, Oct. 1996.

13. HINTZE, H.; CHRISTOFFERSEN, L.; WENZEL, A. In vitro comparison of Kodak Ultra-speed, Ektaspeed, Ektaspeed Plus, and Agfa M2 Comfort dental X-ray films for detection of caries. Oral Surg Oral Med Oral Pathol Oral Radiol Endod, v. 81, n. 2, p. 240-244, Feb. 1996.

14. KERBAUY, W. D.; MORAES, L. C. Processamento digital de imagens de radiografias periapicais subexpostas aos raios X. Rev Odont UNESP, v. 25, n. 2, p. 157-170, jul./dez. 1996. 
TOVO, M. F.; VONO, B. G.; TAVANO, O. Comparação entre filmes radiográficos e o sistema digital Digora, no diagnóstico de lesões de cárie dentinária em superfície proximal de molares decíduos. Pesqui Odontol Bras, v. 14, n. 4, p. 399-405, out./dez. 2000 .

15. KLEIER, D. J.; HICKS, M. J.; FLAITZ, C. M. A comparison of Ultraspeed and Ektaspeed dental X-ray: in vitro study of the radiographic appearance of interproximal lesions. Oral Surg Oral Med Oral Pathol, v. 63, n. 3, p. 381-385, Mar. 1987.

16. McDONNELL, D. Digital dental imaging systems: a review. J Can Dent Assoc, v. 61, n. 11, p. 962-964, Nov. 1995.

17. MOORE, W. S.; DOVE, S. B.; McDAVID, W. D. et al. Comparison of modern imaging systems for the detection of proximal caries. Dentomaxillofac Radiol, v. 27, n. 2, p. 123, Mar. 1998. /Abstract/

18. MOYSTAD, A.; SVANAES, D. B.; RISNES, S. et al. Detection of approximal caries with a storage phosphor system. A comparison of enhanced digital images with dental X-ray film. Dentomaxillofac Radiol, v. 25, n. 4, p. 202-206, Sept. 1996.

19. NIELSEN, L. L.; HOERNOE, M.; WENZEL, A. Radiographic detection of cavitation in approximal surfaces of primary teeth using a digital storage phosphor system and conventional film, and the relationship between cavitation and radiographic lesion depth: an in vitro study. Int J Paediatr Dent, v. 6, n. 3, p. 167-172, Sept. 1996.

20. PORTER, K. H.; von der LEHR, W. H. Diagnosing incipient posterior interproximal caries. Gen Dent, v. 34, n. 2, p. 138-139, Mar./Apr. 1986.

21. RICKETTS, D. N. J.; WHAITES, E. J.; KIDD, E. A. M. et al. An evaluation of the diagnostic yield from bitewing radiographs of small approximal and occlusal carious lesions in a low prevalence sample in vitro using different film types and speeds. Br Dent J, v. 182, n. 2, p. 51-58, Jan. 1997.

22. SVANAES, D. B.; MOYSTAD, A.; RISNES, S. et al. Intraoral storage phosphor radiography for approximal caries detection and effect of image magnification. Oral Surg
Oral Med Oral Pathol Oral Radiol Endod, v. 82, n. 1, p. 94-100, July 1996.

23. SVENSON, B.; LINDVALL, A. M.; GRÖNDAHL, H. G. A comparison of a new dental X-ray film, Agfa Gevaert Dentus M4, with Kodak Ektaspeed and Ultraspeed dental X-ray films. Dentomaxillofac Radiol, v. 22, n. 1, p. 7-12, Feb. 1993.

24. VERDONSCHOT, E. H.; KUJIPERS, J. M. C.; POLDER, B. J. et al. Effects of digital grey-scale modification on the diagnosis of small approximal carious lesions. J Dent, v. 20, n. 1, p. 44-49, Feb. 1992.

25. VERSTEEG, K. H.; SANDERINK, G. C. H.; van der STELT, P. F. Efficacy of digital intra-oral radiography in clinical Dentistry. J Dent, v. 25, n. 3/4, p. 215-224, May/July 1997.

26. VERSTEEG, K. H.; SANDERINK, G. C. H.; VELDERS, X. L. et al. In vivo study of approximal caries depth on storage phosphor plate images compared with dental X-ray film. Oral Surg Oral Med Oral Pathol Oral Radiol Endod, v. 84, n. 2, p. 210-213, Aug. 1997.

27. WENZEL, A. Digital radiography and caries diagnosis. Dentomaxillofac Radiol, v. 27, n. 1, p. 3-11, Jan. 1998.

28. WENZEL, A.; GRÖNDAHL, H. G. Direct digital radiography in the dental office. Int Dent J, v. 45, n. 1, p. 27-34, Jan./Feb. 1995.

29. WENZEL, A.; PITTS, N.; VERDONSCHOT, E. H. et al. Developments in radiographic caries diagnosis. J Dent, v. 21, n. 3, p. 131-140, June 1993.

30. WENZEL, A.; BORG, E.; HINTZE, H. et al. Accuracy of caries diagnosis in digital images from charge-coupled device and storage phosphor systems: an in vitro study. Dentomaxillofac Radiol, v. 24, n. 4, p. 250-254, Nov. 1995.
Recebido para publicação em 26/09/99

Enviado para reformulação em 27/04/00 Aceito para publicação em 24/05/00 